\title{
EFICIÊNCIA NOS MERCADOS FUTUROS AGROPECUÁRIOS BRASILEIROS
}

\author{
Marcos Aurelio Rodrigues * \\ JoÃo Gomes Martines Filho ${ }^{\dagger}$
}

\begin{abstract}
Resumo
Objetivou-se testar a hipótese de passeio aleatório a contratos futuros agropecuários negociados na BM\&FBOVESPA. Refutá-la, significa possível previsibilidade e, por conseguinte, os mercados não seriam fracamente eficientes. Correlações seriais e testes de razão de variância foram utilizados para verificá-las. Os resultados deram suporte à hipótese de passeio aleatório nos mercados futuros de café e da soja, eficientes na forma fraca, e evidências contrárias foram encontradas nos mercados do boi gordo, milho e etanol.
\end{abstract}

Palavras-chave: Razão de variância; eficiência de mercado; commodities.

\begin{abstract}
We aim to test the random walk hypothesis to agricultural future contracts traded at the Brazilian Board of Trade (BM\&FBOVESPA). Refute this hypothesis means possible predictability, therefore these markets are not weakly efficient. We used tests of serial correlation and variance ratio to verify them. Our results do not reject the random walk hypothesis in coffee and soybeans markets but contrary evidences were found for live cattle, corn and ethanol markets.
\end{abstract}

Keywords: Variance ratio; market efficiency; commodities.

JEL classification: G14, Q14

DOI: http://dx.doi .org/10.1590/1413-8050/ea91170

\footnotetext{
* Tribunal de Justiça do Estado do Paraná. Curitiba, Paraná, Brasil. E-mail: rodriguesmarcosaurelio@gmail.com

† Escola Superior de Agricultura "Luiz de Queiroz" - USP. Piracicaba, São Paulo, BRasil. E-mail: martines@usp.br
} 


\section{Introdução}

Inferências sobre predicabilidade dos retornos em finanças possuem importantes implicações práticas e teóricas. Os agentes do agronegócio podem tomar decisões errôneas com respeito ao conjunto de informações incompletas contidas nos preços passados, se os mercados forem não eficientes em sua forma fraca. Tomadas de decisões sujeitas à incompletude informacional implicam custos alocativos ineficientes de seus recursos produtivos.

Nos argumentos de Samuelson (1965) e Fama (1970), mudanças nos preços não podem ser previsíveis se incorporadas totalmente as informações e expectativas de todos os participantes dos mercados. Quanto mais eficiente for o mercado, mais aleatória será a sequência de preços gerados por ele, e o mais eficiente dos mercados será aquele cujas mudanças nos preços sejam completamente aleatórias e imprevisíveis.

Sujeitos a esse comportamento de mercado, investidores não devem esperar lucros que excedam o retorno total de mercado. Em um mercado eficiente, a arbitragem ou possibilidade de lucro livre de risco, com base na diferença de preços sob o mesmo ativo em diferentes mercados, não é possível ser obtida. Estratégias efetuadas levando-se em consideração que os retornos futuros podem ser preditos, somente com base em seu comportamento passado, partem da invalidade da hipótese de eficiência em sua forma fraca, e carteiras de investimento construídas sob modelos de risco retorno dependem do comportamento de passeio aleatório às séries, exemplificando a relevância em estudar o comportamento dos ativos agropecuários negociados na BM\&FBOVESPA.

Embora a literatura internacional seja vasta em estudos que envolvam a análise de eficiência de seus mercados, no Brasil sua maturidade limita-se aos mercados de ativos financeiros. Os estudos sobre os mercados futuros agropecuários são incipientes e pouco explorados metodologicamente (Bitencourt 2007). Bressan \& Leite (2001) fizeram-na com testes de correlação serial, Bitencourt (2007) e Righi \& Ceretta (2011) testaram sob a abordagem de razão de variância. A hipótese conjunta de não enviesamento e eficiência a preços futuros, sob abordagem de cointegração, foi a metodologia mais empregada nos estudos de derivativos agropecuários brasileiros, realizada por Amado \& Carmona (2004), Bitencourt (2007), Duarte et al. (2007), Alves et al. (2008), Moraes et al. (2009), Silva Neto et al. (2010) e Fraga \& Silva Neto (2011).

A contribuição deste estudo está em reexaminar a forma fraca de eficiência no mercado futuro agropecuário brasileiro sob quatro aspectos. Primeiro, quantidade amostral maior que a empregada pela literatura aos contratos de boi gordo, café, etanol, milho e soja. Segundo, avaliar a forma fraca de eficiência nesses mercados com testes de razão de variância individuais propostos por Lo \& MacKinlay (1988) e múltiplos de Chow \& Denning (1993). Terceiro, examinar a hipótese de passeio aleatório nos mercados agropecuários com a recente contribuição de Kim (2006), que propôs o wild bootstrap dos testes individuais e múltiplos. Quarto, considerar a característica díspar de homocedastícia e heterocedastícia presente nas séries diárias dos contratos futuros agropecuários negociados na BM\&FBOVESPA.

O estudo encontra-se estruturado em mais quatro seções além desta introdução: descrevem-se na segunda seção a discussão teórica sobre eficiência de mercado e a literatura empírica sobre eficiência nos mercados de commodities; na terceira seção a metodologia para o teste de hipótese à eficiência fraca nesses mercados; por conseguinte, na quarta seção os resultados empíricos 
encontrados. Evidenciam-se em seguida as considerações finais.

\section{Hipótese da eficiência de mercado}

A hipótese de mercado eficiente remonta ao trabalho de Samuelson (1965), que é creditado por dar respeitabilidade acadêmica à hipótese de passeio aleatório (Lim 2009). Samuelson (1965) elucidou a ideia de mercados eficientes através do seu interesse na precificação temporal de commodities estocáveis. Demonstrou que em um mercado informacionalmente eficiente, as mudanças nos preços devem ser imprevisíveis se forem apropriadamente antecipadas, uma vez que as expectativas e informações de todos os participantes dos mercados são incorporadas completamente. Fama (1970) argumenta que antes de Samuelson (1965), nenhum outro estudo relacionou as teorias de mercados eficientes e passeio aleatório de forma rigorosa, sendo que foi realizado com base na análise dos contratos futuros em mercado de commodities.

O comportamento de caminho aleatório dos preços futuros resulta do perfeito funcionamento dos mercados futuros, sendo definido um mercado futuro perfeito, aquele em que o preço de mercado constitui, em todos os períodos, a melhor estimativa a ser feita, a partir da informação disponível corrente, do preço que será na data de vencimento dos contratos futuros (Working 1962). De acordo com Park \& Irwin (2004), essa definição de mercado futuro perfeito é essencialmente idêntica à de mercado eficiente disposta em Fama (1970), a qual serve como definição padrão na literatura econômica financeira.

Um mercado no qual os preços sempre "refletem completamente" as informações disponíveis é chamado "eficiente" (Fama 1970). Neste estudo, revisouse a literatura teórica e empírica à época e distinguiu-os entre três formas ao teste da hipótese de mercado eficiente, refletido o processo informacional em ordem crescente e sujeitos a subconjuntos de informações relevantes: fraca, semiforte e forte. Na primeira, o conjunto de informações são apenas os preços ou retornos históricos. Na segunda, incluem-se à anterior outras informações publicamente disponíveis. A terceira consiste em todas as informações públicas e, também, quaisquer informações privadas relevantes à formação dos preços que investidores ou grupos detêm.

Uma definição mais recente à eficiência de mercado foi proposta por Jarrow \& Larsson (2012): um mercado $(\mathbb{F}, S)$ é chamado de eficiente ${ }^{1}$ em um horizonte de tempo finito $[0, T]$ com respeito a $\mathbb{F}$ se existe um índice de preço de um bem de consumo $\psi$ e uma economia $\left(\left\{P_{k}\right\}_{k=1}^{K}, \mathbb{F},\left\{\epsilon_{k}\right\}_{k=1}^{K},\left\{U_{k}\right\}_{k=1}^{K}\right)$ para cada $(\psi, S)$ sendo um processo de equilíbrio de preço $S$ em $[0, T]$. Se isso se mantém para todo $T<\infty$, o mercado é chamado de eficiente com respeito a $\mathbb{F}$. Segundo os autores, esse equilíbrio relaciona-se com a noção de equilíbrio de expectativas racionais totalmente revelada sob a forma forte de eficiência de mercado e parcialmente revelada sob a forma fraca de eficiência de mercado.

Segundo Charles \& Darné (2009), a hipótese de passeio aleatório provê uma maneira de testar a forma fraca de eficiência de mercado proposta por Fama (1970) e, consequentemente, não previsibilidade em mercados financeiros. A forma fraca de eficiência de mercado é comumente examinada com a abordagem da hipótese de passeio aleatório (Lim 2009).

\footnotetext{
${ }^{1} \mathbb{F}:=$ filtro de informação; $S:=$ ativo; $P_{k}:=$ finitas crenças dos investidores; $\epsilon:=$ endowment stream; $U:=$ função de utilidade.
} 
As versões de passeio aleatório foram exemplificadas em Campbell et al. (1997). Considere a especificação:

$$
p_{t}=\omega+p_{t-1}+\epsilon_{t},
$$

em que $p_{t}$ representa o logaritmo natural dos preços futuros, $\omega$ um possível drift e $\epsilon_{t}$ o erro. Os três passeios aleatórios de Campbell et al. (1997) diferem em relação às suposições a $\epsilon_{t}$. No passeio aleatório 1 (PA1), o erro de (1) é independente e identicamente distribuído (iid),

$$
\epsilon_{t} \sim \operatorname{iid}\left(0, \sigma^{2}\right),
$$

com média 0 e variância $\sigma^{2}$. No passeio aleatório 2 (PA2), o erro de (1) é independente mas não identicamente distribuído (inid),

$$
\epsilon_{t} \sim \operatorname{inid}\left(0, \sigma^{2}\right)
$$

No passeio aleatório 3 (PA3), o erro de (1) é não independente e não identicamente distribuído (niid),

$$
\epsilon_{t} \sim \operatorname{niid}\left(0, \sigma^{2}\right)
$$

\subsection{Evidências empíricas sobre eficiência nos mercados de commodities}

Os apontamentos de Garcia et al. (1988) a fatores identificados na literatura que potencialmente influenciam a eficiência de mercados futuros agropecuários, dentre eles, as diferentes características das commodities e de seus mercados, o tipo de dados utilizados na análise e a natureza dos testes empregados servem ao delineamento a seção. Portanto, antes das evidências empíricas na literatura, com base nos testes realizados, destacaram-se os estudos segundo suas características, em conjunto com o intervalo amostral, e, em seguida, abordou-se a frequência dos dados utilizados nos estudos.

Devido às características de estocabilidades semelhantes, inerentes a cada commodity, podem ser divididas entre estocáveis e não estocáveis. Os estudos sobre eficiência de mercado no Brasil concentram-se nas mercadorias estocáveis. A análise dos contratos futuros de café foi feita por: Bressan \& Leite (2001) entre 1992 e 1998, Amado \& Carmona (2004) de 1995 a 2003, Bitencourt (2007) de 1996 a 2006 e Cruz Júnior \& Silveira (2007) de 2002 a 2006; de açúcar por Amado \& Carmona (2004) entre 1995 e 2003; de álcool anidro por Alves et al. (2008) entre 2000 e 2006; de milho por Amado \& Carmona (2004) entre 1996 e 2003 e de soja por Duarte et al. (2007) no ano de 2005 e Fraga \& Silva Neto (2011) de 2007 a 2008. A eficiência dos mercados à vista foi verificada somente por Righi \& Ceretta (2011) entre 2006 e 2010, para algodão, café, milho e soja. Apenas dois trabalhos focaram na análise do contrato futuro de boi gordo, commodity não estocável, Moraes et al. (2009) de 2000 a 2004 e Silva Neto et al. (2010) entre 2007 e 2008.

Com respeito à frequência utilizada por estes, houve predominância do uso de séries diárias. Os únicos trabalhos que consideraram frequências diferentes foram Alves et al. (2008), que utilizaram dados semanais, e Bitencourt (2007), que utilizou dados diários, semanais e mensais. Sua justificativa foi relacioná-los a testes de curto, médio e longo prazo ao proporcionar robustez a amostra utilizada. 
Conforme disposto em Lim \& Brooks (2011), testes de correlação serial e análise espectral serviram como as primeiras ferramentas empregadas pela literatura para testar a hipótese de eficiência de mercado em sua forma fraca, de forma pioneira por Fama (1965) e Granger \& Morgenstern (1963), respectivamente.

Bressan \& Leite (2001) testaram se houve autocorrelação nas séries de café no mercado futuro da $\mathrm{BM} \& \mathrm{~F}$, verificado por correlogramas com até cinco defasagens e estatística $Q$ de Ljung \& Box (1978). Conduzidos esses testes para os 31 contratos analisados, no período entre março de 1992 e março de 1998, $42 \%$ deles apresentaram indícios de não eficiência. Sob uma abordagem que buscou verificar a existência de autocorrelação entre os erros de previsão em diferentes horizontes, cinco dias antes do vencimento até noventa dias, variando de cinco em cinco, não houve indícios de ineficiência, pois os coeficientes de autocorrelação foram estatisticamente iguais a zero. Concluíram que o contrato futuro de café foi eficiente no período analisado, sendo que os preços se comportaram como num processo de passeio aleatório.

De acordo com o survey de Lim \& Brooks (2011), a presença de raiz unitária foi utilizada na literatura para testar a forma fraca da hipótese de eficiência de mercado. Os logaritmos dos preços em nível foram não estacionários em estudos revistos por esses autores, assim, os pesquisadores concluíram que os mercados sob estudo apresentaram evidência a favor da hipótese fraca de eficiência.

Apesar de a maioria dos estudos nos mercados agropecuários brasileiros não explicitarem, exceto em Cruz Júnior \& Silveira (2007), a presença de raiz unitária poderia auxiliar na inferência sobre a hipótese de eficiência de mercado em sua forma fraca. Mas Campbell et al. (1997) ressaltam que devido à possibilidade de os $\epsilon$ 's serem processos estacionários com média zero arbitrários, tanto sob a hipótese nula em (1) como sob a hipótese alternativa, o foco desses testes de raiz unitária não é sob a previsibilidade de $p_{t}$. Mesmo sob a hipótese nula, os incrementos de $p_{t}$ podem ser preditos. Conforme seus argumentos, uma vez que existem alternativas ao passeio aleatório sob a hipótese nula de raiz unitária, como, por exemplo, outras formas de dependência serial, esses testes claramente não foram concebidos para detectar a previsibilidade, mas, de fato, são insensíveis por sua construção.

Em resposta ao interesse sobre não estacionariedade nos preços, cointegração e modelos com correção de erro têm sido usados para testar o não enviesamento e eficiência nos mercados futuros (Garcia \& Leuthold 2004). O conceito de não enviesamento é uma versão mais restritiva da forma fraca de eficiência de mercado sugerido por Fama (1970), ao implicar que o preço futuro corrente de uma commodity deve ser igual ao preço esperado no mercado à vista, na data de vencimento do contrato (McKenzie et al. 2002). Uma versão simplista a esse teste é verificar a hipótese nula $\alpha=0$ e $\beta=1 \mathrm{em}$

$$
S_{t}=\alpha+\beta F_{t-1}+\epsilon_{t},
$$

tal que $S$ é o preço à vista, $F$ o preço futuro e $\epsilon$ o erro com média 0 e variância constante. Variações mais complexas a esta foram realizadas, mas a intuição permanece a mesma.

Estudos que analisaram os mercados sob essa abordagem cointegrante ao teste conjunto de eficiência e não eviesamento foram Amado \& Carmona (2004), Bitencourt (2007), Duarte et al. (2007), Alves et al. (2008), Moraes et al. (2009), 
Silva Neto et al. (2010) e Fraga \& Silva Neto (2011). A existência de cointegração às séries à vista e futuras, considerada como condição necessária, mas não suficiente, à eficiência de mercado, foi reportada em todos esses estudos ${ }^{2}$.

Como requisito à hipótese de eficiência de mercado, a hipótese de restrição aos coeficientes $\alpha$ e $\beta[0,1]$ sob teste de razão de verossimilhança, ao vetor cointegrante, atendeu à hipótese conjunta de eficiência de mercado e não enviesamento nos preços futuros de café, no estudo de Bitencourt (2007). Alves et al. (2008) concluíram que o mercado de álcool anidro não atendeu à hipótese de eficiência de mercado pois o teste $\operatorname{sob} \beta=1$ foi rejeitado. Duarte et al. (2007) concluíram que o mercado de soja futuro foi um preditor não enviesado e eficiente em contraposto ao estudo de Fraga \& Silva Neto (2011), pois a hipótese de mercado eficiente para a soja não foi aceita. A restrição conjunta de $\alpha$ e $\beta[0,1]$, imposta ao vetor de cointegração no estudo de Moraes et al. (2009), não foi rejeitada, consequentemente, deu suporte à eficiência e não viés para o mercado de boi gordo. Resultado semelhante ao de Silva Neto et al. (2010), ao sugerirem a não rejeição das hipóteses de mercado eficiente, entretanto evidenciaram a existência de prêmio de risco.

Desde o estudo de Lo \& MacKinlay (1988), os testes de razão da variância emergiram como ferramenta primária para verificar se os retornos das séries acionárias são não correlacionados serialmente (Lim \& Brooks 2011). Lo \& MacKinlay (1988) examinaram os testes de razão de variância propostos por eles, o de raiz unitária sugerido por Dickey \& Fuller $(1979,1981)$, além do teste para correlação serial de Box \& Pierce (1970). Os autores encontraram que os de razão de variância proveem maior poder que os demais sob passeio aleatório heterocedástico. Os estudos de Bitencourt (2007) e Righi \& Ceretta (2011) foram os únicos a utilizarem-nos na inferência sobre a eficiência fraca no mercado futuro de café e nos mercados à vista do algodão, café, milho e soja, respectivamente.

Bitencourt (2007) realizou o teste de razão de variância proposto por Lo \& MacKinlay (1988) para retornos diários, semanais e mensais. Esses apresentaram evidências contrárias à hipótese de passeio aleatório para todas as frequências. A rejeição do passeio aleatório sugeriu que os retornos de curto prazo apresentaram persistência. Além do teste de Lo \& MacKinlay (1988), realizado por Bitencourt (2007), Righi \& Ceretta (2011) utilizaram os testes de Chow \& Denning (1993), Wright (2000) e Chen \& Deo (2006). Os autores concluíram que para todas as commodities se rejeitou a hipótese de passeio aleatório, tanto nos testes que consideram defasagens individualmente, quanto nas estatísticas conjuntas. Isso implicou a rejeição de eficiência de mercado na sua forma fraca aos mercados à vista analisados.

Conforme apontado por Garcia et al. (1988), há divergência nas evidências sobre eficiência nos mercados, devido à natureza dos testes e características dos mercados. Como pôde ser notado, a falta de consenso, tanto metodológico como nos resultados, reforça o objetivo do presente estudo para um maior entendimento com respeito ao comportamento das commodities agropecuárias negociadas na BM\&FBOVESPA.

\footnotetext{
${ }^{2}$ Fraga \& Silva Neto (2011) reportam que na praça de Dourados não houve cointegração com o mercado futuro de soja.
} 


\section{Metodologia}

Estatísticas de portmanteau foram estabelecidas para verificar a hipótese de passeio aleatório 1 de Campbell et al. (1997). Uma vez que essa hipótese implica autocorrelações iguais a zero, as estatísticas $Q$ de Box \& Pierce (1970) e Ljung \& Box (1978), aplicadas aos retornos, fornecem evidências sobre a eficiência dos cinco contratos agropecuários negociados na BM\&FBOVESPA. A análise da hipótese de passeio aleatório 3 de Campbell et al. (1997) foi feita por meio de testes de razão de variância sugeridos por Lo \& MacKinlay (1988) e Chow \& Denning (1993), sob wild bootstrap, seguida a proposta de Kim (2006). O uso de suas versões robustas a heterocedastícia foi escolhido após análise das estatísticas de portmanteau nos resíduos ao quadrado, assim como da estatística dos Multiplicadores de Lagrange, proposta por Engle (1982). Os testes são complementares e, aplicadas as três classes de testes, proveem robustez no estabelecimento das conclusões.

Apesar do desenvolvimento de inúmeros testes estatísticos para verificar a hipótese de passeio aleatório, a classe de testes com base na metodologia da razão de variância tem ganho popularidade nos anos recentes (Charles \& Darné 2009). Segundo seu survey, a metodologia de razão de variância consiste em testar a hipótese de passeio aleatório contra a alternativa de estacionariedade, ao explorar o fato de que a variância dos incrementos de um passeio aleatório é linear em todos os intervalos amostrais, isto é, a variância amostral do retorno no período $k$, de uma série temporal $y_{t}$, é $k$ vezes a variância amostral do retorno de um período.

Seja $y_{t}$ o retorno de uma commodity no tempo $t$, tal que $t=1, \ldots, T$. A razão de variância da $k$-ésima diferença escalonada por $k$, com respeito à variância da primeira diferença, tende a ser igual a um, isto é,

$$
V R(k)=\frac{\sigma^{2}(k)}{\sigma^{2}(1)}
$$

em que $\sigma^{2}(k)$ é $\frac{1}{k}$ a variância da $k$-ésima diferença e $\sigma^{2}(1)$ é a variância da primeira diferença. Sob a hipótese nula de passeio aleatório, $V R(k)$ deve aproximar-se à unidade. Se essa razão for menor do que 1 em longos horizontes, têm-se indícios de correlação serial negativa (reversão à média) e razões maiores do que 1 em horizontes longos indicam correlação serial positiva (aversão à média ou persistência) (Charles \& Darné 2009).

Lo \& MacKinlay (1988) propuseram dois testes estatísticos que exploram a propriedade de (3). Defina o estimador para a variância da $k$-ésima diferença, $\sigma^{2}(k)$, como

$$
\sigma^{2}(k)=\frac{1}{T k} \sum_{t=k}^{T q}\left(y_{t}+\ldots, y_{t-k+1}-k \hat{\mu}\right)^{2}
$$

sendo $\hat{\mu}=\frac{1}{t} \sum_{t=1}^{T} y_{t}$ e o estimador da variância da primeira diferença, $\sigma^{2}(1)$, como

$$
\sigma^{2}(1)=\frac{1}{T} \sum_{t=k}^{T q}\left(y_{t}-\hat{\mu}\right)^{2} .
$$

Os autores mostraram que, sob a suposição de homocedasticidade, então a hipótese nula que $V(k)=1$, a estatística 


$$
M_{1}(k)=\frac{V R(k)-1}{\varphi(k)^{\frac{1}{2}}}
$$

é assintoticamente distribuída como uma $\mathcal{N}(0,1)$, onde

$$
\varphi_{0}(k)=\frac{2(2 k-1)(k-1)}{3 k T} .
$$

Para acomodar a presença de heterocedastícia em $y_{t}$, os autores propuseram uma segunda estatística, robusta sob heterocedasticidade e que segue uma distribuição normal assintótica, definida como:

$$
M_{2}(k)=\frac{V R(k)-1}{\varphi^{*}(k)^{\frac{1}{2}}}
$$

tal que

$$
\varphi^{*}(k)=\sum_{j=1}^{k-1}\left[\frac{2(k-j)}{k}\right]^{2} \delta(j)
$$

e

$$
\delta(j)=\frac{\sum_{t=j+1}^{T}\left(y_{t}-\hat{\mu}\right)^{2}\left(y_{t-j}-\hat{\mu}\right)^{2}}{\left[\sum_{t=1}^{T}\left(y_{t}-\hat{\mu}\right)^{2}\right]^{2}} .
$$

A proposta de Lo \& MacKinlay (1988) verifica a hipótese nula para um $k$ valor individual. Mas a questão central é se as séries temporais revertem à média, requerendo a validade da hipótese nula para todos os valores de $k$. Assim, necessita-se um teste conjunto a múltipla comparação das razões de variância sob diferentes horizontes. Conduzi-los em separado, testando sequencialmente diversos valores de $k$ pode levar a sobre rejeição da hipótese nula e superdimensionamento. Disso, a fraqueza do teste de Lo \& MacKinlay (1988) é ignorar a natureza conjunta do teste à hipótese de passeio aleatório (Charles \& Darné 2009).

Para contornar isso, Chow \& Denning (1993) propuseram um teste múltiplo de razão de variância que requer somente o valor máximo absoluto de $V R(k)$ no conjunto de $m$ testes estatísticos considerados. A estatística é definida por

$$
C D_{1}=\sqrt{T} \max _{1 \leq i \leq m}\left|M_{1}\left(k_{i}\right)\right|
$$

e segue uma distribuição módulo máximo studentizada, com $m$ e $T$ graus de liberdade, $M M S(\alpha, m, T)$. A hipótese nula de passeio aleatório é rejeitada ao nível $\alpha$ de significância se a estatística $M V_{1}$ for maior do que $\left[1-\frac{\alpha^{*}}{2}\right]$-ésimo percentil da distribuição normal, tal que $\alpha^{*}=1-(1-\alpha)^{\frac{1}{m}}$. Entretanto, essa estatística só é válida sob retornos homocedásticos. A versão robusta à heterocedastícia sugerida por Chow \& Denning (1993) pode ser escrita por

$$
C D_{2}=\sqrt{T} \max _{1 \leq i \leq m}\left|M_{2}\left(k_{i}\right)\right|
$$

com os mesmos valores críticos de $C D_{1}$. 
Segundo Kim (2006), os testes de Lo \& MacKinlay (1988) e Chow \& Denning (1993) são assintóticos e podem resultar em deficiências sob pequenas amostras. Kim (2006) propôs como alternativa a esse possível problema desses testes o uso de wild bootstrap, descrito pelo autor como um método de reamostragem que aproxima a distribuição amostral de uma estatística e é aplicável sobre dados com formas desconhecidas de heterocedasticidade condicional e não condicional. Exemplificando o teste com wild bootstrap, embasado em $C D_{2}$, conduzem-se os três seguintes procedimentos:

- formar uma amostra de bootstrap de $T$ observações $y_{t}^{*}=\eta_{t} y_{t}(t=1, \ldots, T)$, tal que $\eta_{t}$ é uma sequência aleatória com $E\left(\eta_{t}\right)=0$ e $E\left(\eta_{t}^{2}\right)=1$;

- calcular $C D_{2}^{*}$, a qual é a estatística da equação (12) das amostragens geradas por bootstrap no primeiro procedimento;

- repetir o primeiro e segundo procedimento $n$ vezes para formar uma distribuição bootstrap $\left\{C D_{2}^{*}(j)\right\}_{j=1}^{n}$ para a estatística do teste.

A distribuição bootstrap $\left\{C D_{2}^{*}(j)\right\}_{j=1}^{n}$ é utilizada para aproximar a distribuição amostral da estatística $C D_{2}$. O valor-p do teste é estimado como uma proporção de $\left\{C D_{2}^{*}(j)\right\}_{j=1}^{n}$ maior do que o valor amostral de $C D_{2} \operatorname{Kim}(2006)$.

\subsection{Base de dados}

A base de dados foi composta pelos preços de fechamento dos contratos ${ }^{3}$ do boi gordo (BGI), milho (CCM), etanol (ETH), café (ICF) e soja (SOJ), mais próximos a vencer, negociados na BM\&FBOVESPA e obtidos no sistema de recuperação de dados desta bolsa. Justifica-se o uso dos contratos mais próximos ao vencimento, pois foram os mais ativos em volume negocial no período em análise. A rolagem do contrato próximo a expirar, para o contrato subsequente, ocorreu no dia anterior ao encerramento de cada contrato.

Tabela 1: Base de dados

\begin{tabular}{|c|c|c|c|}
\hline Ativos - Código & Período & amostral & Número de observações \\
\hline BM\&FBOVESPA & Início & Final & \\
\hline$\overline{\text { Boi gordo - BGI }}$ & 25 de setembro de 2000 & 29 de dezembro de 2011 & 2790 \\
\hline Milho - CCM & 19 de setembro de 2008 & 29 de dezembro de 2011 & 812 \\
\hline Etanol - ETH & 17 de maio de 2010 & 29 de dezembro de 2011 & 406 \\
\hline Café - ICF & 03 de janeiro de 2000 & 29 de dezembro de 2011 & 2972 \\
\hline Soja - SOJ & 27 de agosto de 2004 & 09 de junho de 2011 & 1676 \\
\hline
\end{tabular}

O período escolhido e disposto na Tabela 1 às séries deu-se à disponibilidade desses dados no sistema e também para que fossem mantidas as principais características contratuais do final amostral. $O$ final amostral às séries foi 29 de dezembro de 2011, exceto para o contrato de soja, que se encerrou no dia 09 de junho de 2011.

\footnotetext{
${ }^{3}$ Utilizaram-se seus respectivos códigos na BM\&FBOVESPA para denotá-los.
} 


\section{Resultados e discussões}

Antes de reportar os resultados dos testes, com o intuito de verificar a hipótese de passeio aleatório e, consequentemente, a hipótese de eficiência fraca às commodities em análise, apresentam-se na Tabela 2 as estatísticas descritivas, ressaltando algumas características das séries diárias.

A estatística do teste Jarque \& Bera (1980) indicou rejeição da normalidade às séries desses produtos. Suas distribuições apresentaram comportamento de não normalidade que se relacionam à assimetria e excesso de curtose. Tanto para as séries em nível como às de retorno, para o boi gordo, café e milho apresentaram assimetria à direita e para o etanol e soja assimetria à esquerda. Com relação à curtose, as séries em nível indicaram platicurtose e as de retorno leptocurtose.

Com base nas estatísticas dos testes de Ljung \& Box (1978) e Box \& Pierce (1970) detectou-se forte presença de autocorrelação, tanto para as séries em nível como de retorno, à significância estatística de $1 \%$, exceto nos retornos de milho e soja, que foram estatisticamente significativos a $10 \%$ e $15 \%$, respectivamente. Como o passeio aleatório 1 de Campbell et al. (1997) implica que todas as autocorrelações sejam iguais a zero, dessa autocorrelação nas séries em nível, têm-se as primeiras evidências sobre a hipótese de eficiência de mercado em sua forma fraca, rejeitando-a, conforme os primeiros estudos que a testaram. Entretanto, deve-se ter cautela nessa inferência, pois o estudo de Lo \& MacKinlay (1988) demonstrou que esses testes têm baixo poder, comparados aos de razão de variância, sob passeio aleatório.

Entretanto, resultados obtidos por testes convencionais como estatística F, Box \& Pierce (1970), Ljung \& Box (1978) e Dickey \& Fuller $(1979,1981)$ podem gerar resultados errôneos para verificar um passeio aleatório (Hakkio 1986). Em simulações de Monte Carlo, demonstrou que esses testes têm baixo poder relativo, pois não distinguem entre um passeio aleatório e algo próximo a um passeio aleatório.

Seguido o critério de Tsay (2005), para a seleção de defasagens ao teste de Ljung \& Box (1978), $\ln (T)$, as estatísticas de Ljung \& Box (1978) e Box \& Pierce (1970), às séries ao quadrado, assim como o teste dos multiplicadores de Lagrange de Engle (1982), sugerem existência de heterocedastícia nos preços em nível e de retorno a três contratos: boi gordo, café e soja. Ressalta sua não presença nos retornos de milho e etanol, fato estilizado esperado em séries financeiras diárias.

A presença de autocorrelações indicadas na Tabela 3 sugere razões de variância diferentes à unidade, confirmadas na Figura 1. Nela dispõem-se as estimativas das razões de variância até 8 lags com intervalo de confiança a $95 \%$.

Em um mercado eficiente, espera-se que as razões de variância sejam próximas à unidade. Entretanto, apenas para a série do café isso ocorre de forma clara. Com o aumento do horizonte temporal, houve indícios de reversão à média. Reversão à média também ocorreu no caso do etanol, mas com um horizonte de tempo maior, comparado ao contrato de café, logo, ambos serialmente correlacionados negativamente. Nas demais séries, o comportamento das razões de variância apresentou valores crescentes e superiores à unidade, característico de aversão à média, demonstrando crescimento da variância mais do que proporcional com o tempo. Isso sugere que os retornos dessas séries possuíram presença dominante de autocorrelações positivas. 
Tabela 2: Estatísticas descritivas

\begin{tabular}{|c|c|c|c|c|c|c|c|c|c|c|}
\hline & \multicolumn{2}{|c|}{ BGI } & \multicolumn{2}{|c|}{ CCM } & \multicolumn{2}{|c|}{ ETH } & \multicolumn{2}{|c|}{ ICF } & \multicolumn{2}{|c|}{ SOJ } \\
\hline & $\ln$ & $\nabla \ln$ & $\ln$ & $\nabla \ln$ & $\ln$ & $\nabla \ln$ & $\ln$ & $\nabla \ln$ & $\ln$ & $\nabla \ln$ \\
\hline Observações & 2790 & 2789 & 812 & 811 & 406 & 405 & 2972 & 2971 & 1676 & 1675 \\
\hline Mínimo & 3,6243 & $-0,1049$ & 2,8758 & $-0,0980$ & 6,5827 & $-0,3352$ & 3,7136 & $-0,1522$ & 2,3933 & $-0,1616$ \\
\hline Máximo & 4,7532 & 0,0862 & 3,4819 & 0,0917 & 7,4413 & 0,0629 & 5,9839 & 0,1474 & 3,5779 & 0,0488 \\
\hline Média & 4,1442 & 0,0003 & 3,1628 & 0,0002 & 7,0086 & 0,0013 & 4,7530 & 0,0003 & 2,9718 & 0,0005 \\
\hline Desvio Padrão & 0,2798 & 0,0097 & 0,1794 & 0,0136 & 0,1847 & 0,0240 & 0,5243 & 0,0205 & 0,3213 & 0,0153 \\
\hline Assimetria & 0,2534 & 0,0586 & 0,1517 & 0,0379 & $-0,5625$ & $-9,7788$ & 0,1840 & 0,3371 & $-0,0552$ & $-1,3296$ \\
\hline Curtose & $-0,9585$ & 13,3671 & $-1,4347$ & 9,1537 & $-0,2118$ & 127,1725 & $-0,4875$ & 5,8234 & $-1,4006$ & 11,4595 \\
\hline Jarque-Bera & 136,36 & 20802,05 & 72,39 & 2850,18 & 22,23 & 282195,95 & 46,01 & 4262,98 & 137,47 & 9687,17 \\
\hline Valor-p & 0,0000 & 0,0000 & 0,0000 & 0,0000 & 0,0000 & 0,0000 & 0,0000 & 0,0000 & 0,0000 & 0,0000 \\
\hline
\end{tabular}




\begin{tabular}{|c|c|c|c|c|c|c|c|c|c|c|}
\hline & \multicolumn{2}{|c|}{ BGI } & \multicolumn{2}{|c|}{$\mathrm{CCM}$} & \multicolumn{2}{|c|}{ ETH } & \multicolumn{2}{|c|}{ ICF } & \multicolumn{2}{|c|}{ SOJ } \\
\hline & $\ln$ & $\nabla \ln$ & $\ln$ & $\nabla \ln$ & $\ln$ & $\nabla \ln$ & $\ln$ & $\nabla \ln$ & $\ln$ & $\nabla \ln$ \\
\hline Ljung-Box & 22112,99 & 64,57 & 5536,93 & 11,77 & 2581,76 & 24,51 & 23705,38 & 31,72 & 13234,17 & 12,01 \\
\hline Valor-p & 0,0000 & 0,0000 & 0,0000 & 0,1084 & 0,0000 & 0,0009 & 0,0000 & 0,0001 & 0,0000 & 0,1505 \\
\hline Ljung-Box ${ }^{2}$ & 22125,46 & 33,31 & 5535,72 & 5,88 & 2577,10 & 0,25 & 23702,60 & 1880,38 & 13240,93 & 69,53 \\
\hline Valor-p & 0,0000 & 0,0001 & 0,0000 & 0,5536 & 0,0000 & 1,0000 & 0,0000 & 0,0000 & 0,0000 & 0,0000 \\
\hline Box-Pierce & 22061,63 & 64,47 & 5496,27 & 11,67 & 2544,40 & 24,11 & 23653,66 & 31,66 & 13183,04 & 11,98 \\
\hline Valor-p & 0,0000 & 0,0000 & 0,0000 & 0,1118 & 0,0000 & 0,0011 & 0,0000 & 0,0001 & 0,0000 & 0,1522 \\
\hline Box-Pierce ${ }^{2}$ & 22074,08 & 33,22 & 5495,07 & 5,85 & 2539,81 & 0,25 & 23650,90 & 1876,71 & 13189,78 & 69,23 \\
\hline Valor-p & 0,0000 & 0,0001 & 0,0000 & 0,5572 & 0,0000 & 1,0000 & 0,0000 & 0,0000 & 0,0000 & 0,0000 \\
\hline ML-ARCH & 2778,64 & 29,64 & 799,39 & 3,90 & 390,65 & 0,35 & 2959,71 & 574,95 & 1663,96 & 37,55 \\
\hline Valor-p & 0,0000 & 0,0002 & 0,0000 & 0,8657 & 0,0000 & 1,0000 & 0,0000 & 0,0000 & 0,0000 & 0,0000 \\
\hline $\ln (T)^{1}$ & 8,0000 & & 7,0000 & & 7,0000 & & 8,0000 & & 8,0000 & \\
\hline
\end{tabular}




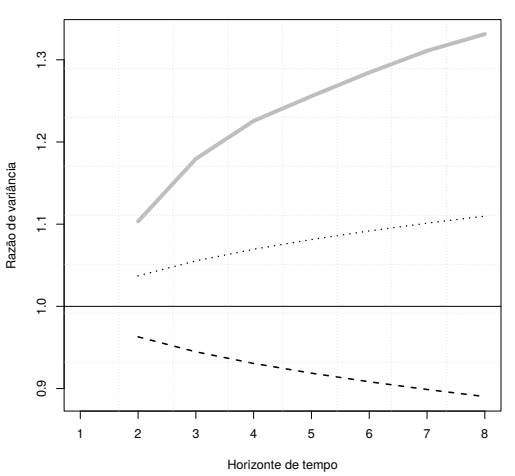

(a) $\nabla \ln$ BGI

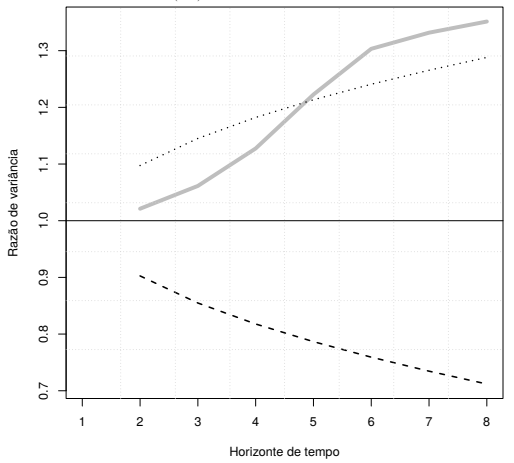

(c) $\nabla \ln \mathrm{ETH}$

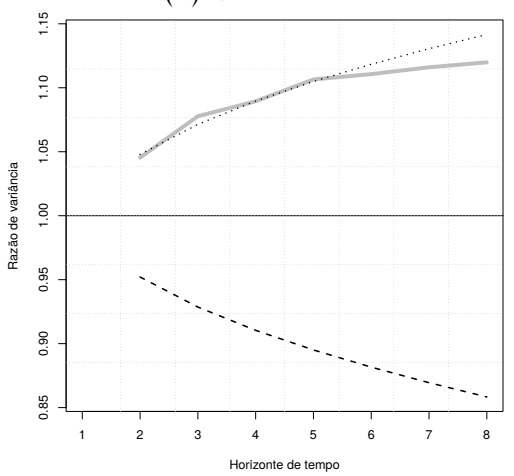

(e) $\nabla \ln \mathrm{SOJ}$

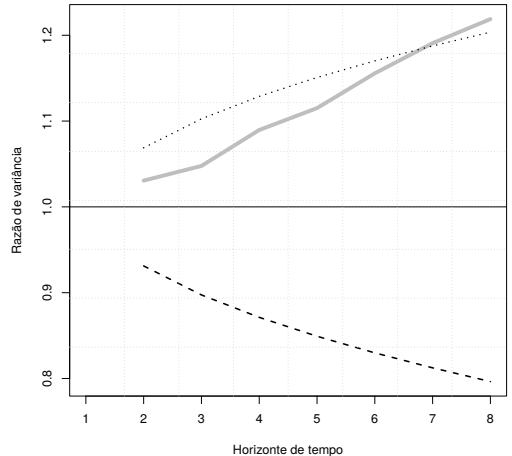

(b) $\nabla \ln \mathrm{CCM}$

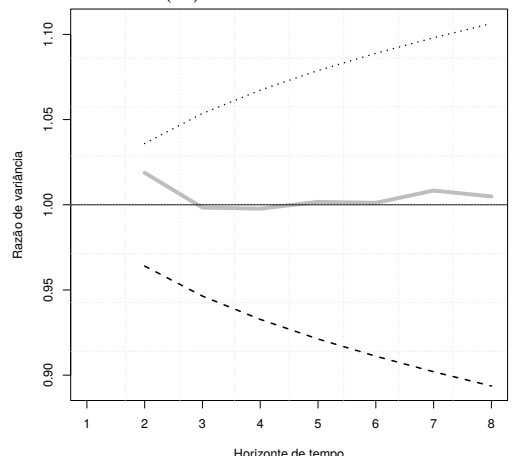

(d) $\nabla \ln$ ICF

Fonte: Dados da Pesquisa.

Figura 1: Razões de variância 
Têm-se indícios que os mercados de boi gordo, milho e etanol, por estarem fora do intervalo de confiança às razões de variância empíricas, não sejam eficientes, e nos mercados de café e soja há evidência à eficiência. Com base nessas razões parte-se para o cálculo dos testes de razões de variância.

Tabela 4: Razões de variância com wild bootstrap proposto por Kim (2006) ao teste de Lo \& MacKinlay (1988) sob os retornos

\begin{tabular}{lcrrrrr}
\hline Teste & $k$ & $\nabla \ln$ BGI & $\nabla \ln$ CCM & $\nabla \ln$ ETH & $\nabla \ln$ ICF & $\nabla \ln$ SOJ \\
\hline$M_{1}$ & 2 & & 0,8716 & 0,4257 & & \\
Valor-p & & & 0,5260 & 0,8740 & & \\
$M_{1}$ & 4 & & 1,3638 & 1,3712 & & \\
Valor-p & & & 0,2730 & 0,2410 & & \\
$M_{1}$ & 6 & & 2,1066 & 2,3908 & & \\
Valor-p & & & 0,0600 & 0,0140 & & \\
$M_{1}$ & 8 & & 2,1325 & $-0,3178$ & & \\
Valor-p & & & 0,0200 & 0,8540 & & \\
\hline$M_{2}$ & 2 & 4,6618 & 0,6823 & 0,2956 & 0,5510 & 1,4743 \\
Valor-p & & 0,0000 & 0,5260 & 0,8740 & 0,6170 & 0,1210 \\
$M_{2}$ & 4 & 5,3052 & 1,1130 & 1,1524 & $-0,0355$ & 1,5394 \\
Valor-p & & 0,0000 & 0,2730 & 0,2410 & 0,9750 & 0,1060 \\
$M_{2}$ & 6 & 4,8412 & 1,8024 & 2,1311 & 0,0501 & 1,2554 \\
Valor-p & & 0,0000 & 0,0600 & 0,0140 & 0,9640 & 0,1740 \\
$M_{2}$ & 8 & 3,0594 & 1,9081 & $-0,2650$ & $-0,1409$ & 0,8765 \\
Valor-p & & 0,0010 & 0,0200 & 0,8540 & 0,8980 & 0,3510 \\
\hline Fonte: Dados da Pesquisa & & & & \\
\hline
\end{tabular}

Conforme demonstrado na Tabela 3, os retornos do boi gordo, café e soja apresentaram heterocedastícia, logo, apenas os testes robustos à heterocedasticidade foram reportados. De acordo com os testes individuais de Lo \& MacKinlay (1988) sob wild bootstrap de Kim (2006) expostos na Tabela 4, a hipótese nula de um passeio aleatório foi rejeitada para os retornos do boi gordo. Isso evidencia poder de previsão para horizontes de curto prazo, entre 2 e 8 períodos, invalidando a hipótese fraca de eficiência de mercado, representada pelo modelo de passeio aleatório 3 de Campbell et al. (1997). Invalidades às hipóteses também ocorrem nos mercados de milho e etanol, mas em horizontes mais longos, 6 e 8 dias. Pode-se inferir que os mercados de café e soja foram eficientes na forma fraca, nos períodos analisados, devido à não rejeição da hipótese de caminho aleatório, aos níveis tradicionais de significância estatística.

Possibilidades de previsões discrepantes, em horizontes diferentes, impossibilitaram a unicidade das inferências sobre a hipótese de caminho aleatório. Para contornar esse problema, parte-se ao teste conjunto entre todos os horizontes, proposto por Chow \& Denning (1993).

Com base na metodologia múltipla do teste de Chow \& Denning (1993) sob wild bootstrap de Kim (2006), a hipótese nula à razão de variância igual a 1 foi rejeitada nos mercados em que o teste de Lo \& MacKinlay (1988) foi inconclusivo. Assim, os resultados dos testes individuais reforçam o resultado do teste múltiplo e indicam eficiência de mercado na forma fraca apenas aos mercados do café e da soja.

As evidências desfavoráveis à eficiência possuem implicações nos estudos sobre hedge desses mercados. De acordo com Rodrigues \& Alves (2010), nos 
Tabela 5: Razões de variância com wild bootstrap proposto por Kim (2006) ao teste de Chow \& Denning (1993) sob os retornos

\begin{tabular}{lccccc}
\hline Teste & $\nabla \ln$ BGI & $\nabla \ln$ CCM & $\nabla \ln$ ETH & $\nabla \ln$ ICF & $\nabla \ln$ SOJ \\
\hline$C D_{1}$ & & 2,1325 & 2,3908 & & \\
Valor-p & & 0,0570 & 0,0350 & & \\
$C D_{2}$ & 5,3052 & 1,9081 & 2,1311 & 0,5510 & 1,5394 \\
Valor-p & 0,0000 & 0,0570 & 0,0350 & 0,8870 & 0,2330 \\
\hline
\end{tabular}

Fonte: Dados da Pesquisa.

estudos empíricos brasileiros sobre razão e efetividade de hedge dos mercados agropecuários há predominância no uso da função de utilidade média variância como arcabouço, assim como baixa efetividade do hedge. Segundo demonstrações ${ }^{4}$ de Kroner \& Sultan (1993), esse arcabouço pressupõe que os retornos nos mercados futuros sigam um processo martingal - por conseguinte, eficientes na forma fraca - para que a razão de hedge seja ótima. Assim, pode-se atribuir a baixa efetividade de hedge nos estudos empíricos brasileiros sobre os mercados agropecuários à não eficiência dos mercados futuros, o que causa a não otimalidade da razão de hedge.

O funcionamento informacional nos mercados cuja hipótese de passeio aleatório não foi rejeitada, e, portanto, eficientes na forma fraca, auxiliam os agentes desses mercados na descoberta de preços. Assim, esses mercados futuros eficientes contribuem para as tomadas de decisões sobre a produção, comercialização e estocagem (Garcia et al. 1988). Entretanto, nos mercados em que a hipótese nula de passeio aleatório foi rejeitada, os agentes estão sujeitos às informações errôneas indicadas nos mercados futuros dos ativos. Isso ocasiona incompletude informacional e, segundo Garcia et al. (1988), possível redução do excedente econômico, devido à possível alocação equivocada dos recursos desses agentes.

Conforme argumentos de Garcia et al. (1988), as diferentes características das commodities e de seus mercados influenciam a eficiência dos mercados futuros agropecuários. Nesse sentido, particularidades desses mercados, dispostas na Tabela 6, como liquidez, custos de transação e o poder de mercado dos participantes podem auxiliar na explicação das rejeições e não rejeições à hipótese nula.

Observa-se que o ativo com a maior média diária de contratos negociados foi o café, sendo que neste ativo as evidências obtidas com os testes de razão de variância indicaram não rejeição à hipótese de eficiência de mercado. Note que Amado \& Carmona (2004) encontraram evidências favoráveis a eficiência do mercado de café e indicaram a liquidez como fator para esse resultado.

A discrepância entre a evidência sugerida nos testes de razão de variância no mercado de soja e sua liquidez indica que o número de contratos negociados não corresponde como fator explicativo, haja vista sua ínfima liquidez comparada a outros ativos. Note que no survey de Garcia \& Leuthold (2004), os estudos no mercado norte-americano da soja indicam eficiência deste mercado. Portanto, um possível fator que pode explicar a eficiência nesse mercado

\footnotetext{
${ }^{4}$ Para maiores informações, ver a primeira nota de rodapé de Kroner \& Sultan (1993), assim como as passagens das equações (5-6) e (9-10), as quais são simplificadas com base no pressuposto de o mercado futuro seguir um processo martingal.
} 
Tabela 6: Características dos mercados

\begin{tabular}{|c|c|c|c|c|c|}
\hline & BGI & $\mathrm{CCM}$ & ETH & ICF & SOJ \\
\hline \multicolumn{6}{|l|}{ Liquidez } \\
\hline Média d & 755 & 519 & 45 & 996 & 87 \\
\hline$\%$ de $\log$ retornos $=0$ & 3,22 & 3,94 & 9,62 & 1,64 & 12,47 \\
\hline \multicolumn{5}{|c|}{ Tipos de participantes dos contratos em aberto } & $(\%)$ \\
\hline Pess & 5,9578 & 6,8968 & 2,2898 & 4,7176 & 2,0967 \\
\hline Investidor Institucional & 19,5215 & 3,0077 & 0,7255 & 10,2125 & 2,2898 \\
\hline Investidores Não Residentes & 7,2892 & 1,9100 & 3,3574 & 25,2093 & 1,4342 \\
\hline Pessoa Jurídica Não Financeira & 34,8054 & 72,1241 & 84,3073 & 51,9801 & 85,6676 \\
\hline Pes & 32,4271 & 16,0614 & 9,3200 & 7,8804 & 8,5116 \\
\hline Coeficiente de Gini & 0,3313 & 0,6139 & 0,6968 & 0,4474 & 0,6995 \\
\hline \multicolumn{6}{|c|}{$\begin{array}{l}\text { Fonte: Sistema de recuperação de dados da BM\&FBOVESPA. } \\
\text { Nota: O período compreende o disposto na Tabela 1. A média diária de contratos } \\
\text { negociados e o percentual de log retornos iguais a zero se referem ao primeiro } \\
\text { vencimento. Devido à disponibilidade no sistema de recuperação de dados da } \\
\text { BM\&FBOVESPA, os percentuais de contratos em aberto por tipo de participante } \\
\text { referem-se a todos os vencimentos. }\end{array}$} \\
\hline
\end{tabular}

brasileiro é a formação de seus preços ser dada nos mercados internacionais, repercutindo no mercado brasileiro.

Os contratos de boi gordo e milho apresentaram médias de contratos negociados inferiores ao do café, enquanto que os testes indicaram rejeição à eficiência. Ademais, liquidez discrepante no etanol e soja revela que esses foram os menos líquidos no período de análise, sendo que houve evidência não favorável à eficiência no mercado da soja. Assim, apesar de Garcia et al. (1988), Amado \& Carmona (2004), Gilson \& Kraakman (2014), entre outros, indicarem a liquidez dos contratos para que esses sejam informacionalmente eficientes, possíveis explicações das não rejeições e rejeições à hipótese de eficiência de mercado não podem ser centradas apenas nesse indicativo.

Como consequência da não eficiência nos mercados futuros agropecuários brasileiros, os preços não respondem tão rapidamente às informações públicas contidas em seus preços passados, de forma que os investidores podem obter lucros por arbitragem ao negociar tal informação (Gilson \& Kraakman 2014). Dois mecanismos podem explicar tal resposta às informações para os autores. Primeiro, pode-se considerar que os profissionais desses mercados não compreendem tão rapidamente as novas informações em um curto espaço de tempo, quando comparado aos mercados eficientes. Segundo, nos mercados eficientes, considera-se que praticamente todos os profissionais atuantes no mercado possuem aprendizado às novas informações quase simultaneamente. No entanto, a velocidade com que as informações refletem nos preços é função da liquidez do mercado. Os autores notam que esses mecanismos informacionais sob os preços dependem dos custos de obtenção das informações e dos custos de arbitragem - isto é - do custo de negociar tais informações.

Segundo Lesmond et al. (1999), um ativo com elevados custos de transação tem movimentos dos preços menos frequentes e maior frequência de retornos iguais a zero comparado a um ativo com baixos custos de transação. Utilizando-se o percentual de retornos iguais a zero, proxy sugerida pelos autores para estimar os custos de transação, observam-se elevados custos de transação nos mercados de etanol e de soja ao serem comparados aos demais. Assim como os custos de transação, a baixa liquidez dos contratos dificulta a 
operacionalização das possíveis arbitragens existentes nos mercados não eficientes. Além disso, conforme McKenzie et al. (2002), o baixo nível de negócios dificulta a potencial descoberta de preços aos agentes do sistema de comercialização.

Assim como no mercado da soja, os mercados de etanol e milho apresentaram maior concentração dos contratos em aberto por tipo de participantes. Essa concentração, mensurada pelo coeficiente de Gini, possibilita inferir que há poder de mercado nas negociações desses ativos, em particular das pessoas jurídicas não financeiras. Ao contrário do que ocorre nos mercados de café e boi gordo, a não atração dos diversos tipos de participantes nesses mercados, dificulta a eficiência informacional nesses mercados. Ressalta-se que apesar de a menor concentração dos participantes no mercado de boi gordo ser favorável à eficiência desse mercado, Urso (2007) sugere existência de poder de mercado na aquisição de bois pelos frigoríficos no mercado à vista. Segunda a autora, a estrutura dessa indústria é de uma produção pecuária pulverizada, um número elevado de frigoríficos, mas que já apresenta sinais de concentração, e passa a se estruturar como um oligopólio. Os resultados encontrados em seu estudo corroboraram a visão de que os frigoríficos - em sua maioria associados a pessoas jurídicas não financeiras — têm mais informação, no mercado futuro, que os demais agentes.

Os resultados não condizentes com a eficiência dos mercados de milho e etanol também podem ser creditados às intervenções governamentais na estrutura de preços desses mercados. As intervenções governamentais, sugeridas como fator de influência ao bom funcionamento dos mercados no survey de Garcia et al. (1988), e utilizadas como justificativa no estudo sobre a eficiência do mercado de álcool anidro por Alves et al. (2008), têm influência adversa à eficiência informacional. Assim, devem-se considerar atenuações no grau dessas intervenções como forma de obter eficiência alocativa de recursos aos agentes dessa cadeia por meio do mercado futuro.

\section{Considerações finais}

Os resultados demonstraram que, no intuito de retornos especulativos, por mais que se tentem fazer previsões para essas séries, a evidência a favor de eficiência dos mercados de café e da soja indica que são imprevisíveis.

Os resultados da rejeição à hipótese de passeio aleatório aos retornos sugerem presença de predicabilidade nos mercados futuros agropecuários de boi gordo, milho e etanol. Refutá-la indica que os investimentos em ativos desses mercados podem proporcionar a agentes - investidores, arbitradores, especuladores e gestores financeiros - retornos superiores à média do mercado.

Desses resultados, as imperfeições existentes podem ocasionar a não proteção necessária a hedgers ao utilizarem contratos futuros que não refletem todas as informações existentes no mercado em seus preços passados. Isso dificulta a operacionalização de estratégias, com objetivo de redução de risco, embasadas na historicidade de suas cotações. A não eficiência fraca desses mercados pode explicar a baixa efetividade do hedge nesses ativos.

Do lado de eficiência alocativa necessária a agentes que fazem uso dos contratos nos mercados de commodities fracamente não eficientes, os mecanismos de apreçamento não asseguram alocação eficiente dos recursos aos agentes, com efeitos negativos para a cadeia dependente desse ativo. A ineficiência 
evidenciada pode levar os membros que regulamentam e normatizam esses contratos negociados na BM\&FBOVESPA a tomarem medidas necessárias à sua correção e que elevem o volume negociado desses ativos, indispensável para que ocorra maior aleatoriedade, com possível redesenho contratual e formadores de mercado.

A rejeição da hipótese de eficiência nesses mercados oferece evidência de que ainda são ineficientes, e não uma evidência que o arcabouço teórico, no qual a hipótese se baseia, pode estar equivocado.

\section{Agradecimentos}

Os autores agradecem aos comentários e sugestões do editor, dos pareceristas anônimos, assim como de: Alexandre Florindo Alves, José Cesar Cruz Júnior, Fabio Lanhoso de Mattos, Márcio Poletti Laurini e Rodrigo Lanna Franco da Silveira.

\section{Referências Bibliográficas}

Alves, J. S., Duarte, G. S. \& Lima, R. C. (2008), 'Teste da eficiência do mercado futuro do álcool anidro no brasil: uma análise de co-integração', Revista Econômica do Nordeste 39(1), 173-184.

Amado, C. F. P. \& Carmona, C. U. M. (2004), 'Uma análise da eficiência dos mercados futuros agrícolas brasileiros', IV Encontro Brasileiro de Finanças, Rio de Janeiro.

Bitencourt, W. A. (2007), 'Ensaios empíricos sobre a eficiência do mercado futuro de café', Departamento de Administração e Economia, Universidade Federal de Lavras, Mestrado em administração.

Box, G. E. P. \& Pierce, D. A. (1970), 'Distribution of residual autocorrelations in autoregressive-integrated moving average time series models', Journal of the American Statistical Association 65(332), 1509-1526.

Bressan, A. A. \& Leite, C. A. M. (2001), 'Eficiência do mercado futuro de café no brasil', Reuna 6(1), 11-32.

Campbell, J. Y., Lo, A. W. \& MacKinlay, A. C. (1997), The econometrics of financial markets, Princeton University Press, Princeton.

Charles, A. \& Darné, O. (2009), 'Variance-ratio tests of random walk: an overview', Journal of Economic Surveys 23(3), 503-527.

Chen, W. W. \& Deo, R. S. (2006), 'The variance ratio statistic at large horizons', Econometric Theory 22(2), 206-234.

Chow, K. \& Denning, K. C. (1993), 'A simple multiple variance ratio test', Journal of Econometrics 58(3), 385-401.

Cruz Júnior, J. C. \& Silveira, R. L. F. (2007), 'Análise de eficiência, cointegração e exogeneidade nos mercados futuros de café na bm\&f, nyb ot e liffe', XLV Congresso Brasileiro de Economia e Sociologia Rural. 
Dickey, D. A. \& Fuller, W. A. (1979), 'Distribution of the estimators for autoregressive time series with a unit root', Journal of the American Statistical Association 74(366), 427-431.

Dickey, D. A. \& Fuller, W. A. (1981), 'Likelihood ratio statistics for autoregressive time series with a unit root', Econometrica 49(4), 1057-1072.

Duarte, G. B., Lima, R. C. \& Alves, J. S. (2007), 'Co-integração e eficiência do mercado futuro da soja no brasil', Congresso Brasileiro de Economia e Sociologia Rural, Londrina.

Engle, R. F. (1982), 'Autoregressive conditional heteroscedasticity with estimates of the variance of united kingdom inflation', Econometrica 50(4), 9871007.

Fama (1970), 'Efficient capital markets: a review of theory and empirical work', The Journal of Finance 25(2), 383-417.

Fama, E. F. (1965), 'The behavior of stock-market prices', The Journal of Business 38(1), 34-105.

Fraga, G. J. \& Silva Neto, W. A. (2011), 'Eficiência no mercado futuro de commodity: evidências empíricas', Revista Econômica do Nordeste 42(1), 125137.

Garcia, P., Hudson, M. A. \& Waller, M. L. (1988), 'The pricing efficiency of agricultural futures markets: an analysis of previous research results', Southern Journal of Agricultural Economics 20(1), 119-130.

Garcia, P. \& Leuthold, R. M. (2004), 'A selected review of agricultural commodity futures and options markets', European Review of Agricultural Economics 31(3), 235-272.

Gilson, R. J. \& Kraakman, R. (2014), 'Market efficiency after the financial crisis: it's still a matter of information costs', Virginia Law Review 100(2), 313375.

Granger, C. W. J. \& Morgenstern, O. (1963), 'Spectral analysis of new york stock market prices', Kyklos 16(1), 1-27.

Hakkio, C. (1986), 'Does the exchange rate follow a random walk? a monte carlo study of four tests for a random walk', Journal of International Money and Finance 5(2), 221-229.

Jarque, C. M. \& Bera, A. K. (1980), 'Efficient tests for normality, homoscedasticity and serial independence of regression residuals', Economics Letters 6(3), 255-259.

Jarrow, R. A. \& Larsson, M. (2012), 'The meaning of market efficiency', Mathematical Finance 22(1), 1-30.

Kim, J. H. (2006), 'Wild bootstrapping variance ratio tests', Economics Letters 92(1), 38-43.

Kroner, K. F. \& Sultan, J. (1993), 'Time-varying distributions and dynamic hedging with foreign currency futures', The Journal of Financial and Quantitative Analysis 28(4), 535-551. 
Lesmond, D., Ogden, J. \& Trzcinka, C. (1999), 'A new estimate of transaction costs', Review of Financial Studies 12(5), 1113-1141.

Lim, K. (2009), 'An empirical analysis of the weak-form efficiency of stock markets', Department of Econometrics and Business Statistics, Monash University, Doctor of Philosophy.

Lim, K. \& Brooks, R. (2011), 'The evolution of stock market efficiency over time: a survey of the empirical literature', Journal of Economic Surveys 25(1), 69-10.

Ljung, G. M. \& Box, G. E. P. (1978), 'On a measure of lack of fit in time series models', Biometrika 65(2), 297-303.

Lo, A. W. \& MacKinlay, A. C. (1988), 'Stock market prices do not follow random walks: evidence from a simple specification test', Review of Financial Studies 1(1), 41-66.

McKenzie, A. M., Jiang, B., Djunaidi, H., Hoffman, L. A. \& Wailes, E. J. (2002), 'Unbiasedness and market efficiency tests of the u.s. rice futures market', Review of Agricultural Economics 24(2), 474-493.

Moraes, A. S., Lima, R. C. \& de Souza Melo, A. (2009), 'Análise da eficiência do mercado futuro brasileiro de boi gordo usando co-integração', Revista de Economia e Sociologia Rural 47(3), 601-614.

Park, C.-H. \& Irwin, S. H. (2004), 'The profitability of technical analysis: a review', AgMAS Project Research Reports, n. 04.

Righi, M. B. \& Ceretta, P. S. (2011), 'Previsibilidade e eficiência no mercado agrícola', Ciência Rural 41(10), 1844-1850.

Rodrigues, M. A. \& Alves, A. F. (2010), 'Efetividade e razão ótima de hedge: um survey', XLVIII Congresso Brasileiro de Economia e Sociologia Rural, Campo Grande.

Samuelson, P. A. (1965), 'Proof that properly anticipated prices fluctuate randomly', Industrial Management Review 5(2), 41-49.

Silva Neto, W. A., Fraga, G. J. \& Marques, P. V. (2010), 'Eficiência de mercado: evidências empíricas para os preços spot e futuro de boi gordo', Revista de Economia 36(3), 7-24.

Tsay, R. S. (2005), Analysis of financial time series, 2 edn, John Wiley \& Sons.

Urso, F. S. P. (2007), 'A cadeia da carne bovina no brasil: uma análise de poder de mercado e teoria da informação', Escola de Economia de São Paulo, Fundação Getúlio Vargas - Doutorado em Economia de Empresas.

Working, H. (1962), 'New concepts concerning futures markets and prices', The American Economic Review 52(3), 431-459.

Wright, J. H. (2000), 'Alternative variance-ratio tests using ranks and signs', Journal of Business \& Economic Statistics 18(1), 1-9. 